This is the final peer-reviewed accepted manuscript of:

Bagnoli, L., \& Negroni, G. (2019). Egalitarianism: An evolutionary perspective. Metroeconomica, 70(1), 24-44.

The final published version is available online at:

https://doi.org/10.1111/meca.12225

Rights / License:

The terms and conditions for the reuse of this version of the manuscript are specified in the publishing policy. For all terms of use and more information see the publisher's website.

This item was downloaded from IRIS Università di Bologna (https://cris.unibo.it/)

When citing, please refer to the published version. 
This is the accepted version of the following article:

Bagnoli L, Negroni G. Egalitarianism: An evolutionary perspective.

Metroeconomica. 2019; 70: 24-44. https://doi.org/10.1111/meca.12225

\title{
Egalitarianism. An evolutionary perspective
}

\author{
Lidia Bagnoli $^{1}$, Giorgio Negroni ${ }^{2}$ \\ Ministry of Economy and Finance \\ and \\ University of Bologna
}

\begin{abstract}
Two parties bargain over a pie, the size of which is determined by their previous investment decisions. Investment costs are heterogenoeus. The bargaining rule is sensitive to investment behavior. Our main result is that egalitarianism is more likely to emerge in situations in which private property over the fruits of one's own independent investment is not secured.
\end{abstract}

Key Words: evolution; social norms; stochastically stable equilibrium; egalitarianism; inequality; Rawls.

JEL Codes. C78, D83, L14, Z13.

\section{Introduction}

Many relevant economic problems are related to a simple two-stage game in which a pie has to be firstly generated by the investment decision of some agent; then, after the investment is made, bargaining determines the division of the pie. An inefficient equilibrium is observed when the investing party, expecting to receive a share not covering the investment cost, keeps from investing. This is the so-called "hold-up problem".

\footnotetext{
${ }^{1}$ Permanent Address: Ministry of Economy and Finance, via Solferino 11, 47121 Forlì, Italy. E-mail: lidia.bagnoli@tesoro.it

${ }^{2}$ Corresponding author. Department of Economics, University of Bologna, Strada Maggiore 45, 40125 Bologna, Italy. E-mail: giorgio.negroni@unibo.it
} 
Evolutionary dynamics in models with advance production and successive bargaining have been studied by Troger (2002), Ellingsen and Robles (2002) and Dawid and MacLeod (2001, 2008). Broadly speaking this literature has shown that evolution may or may not support efficiency depending on whether only one (Troger, 2002; Ellingsen and Robles, 2002) or both parties (Dawid and MacLeod, 2001, 2008) can invest in the project, respectively. This literature shares the common assumption that investment costs are the same for all investing agents. Andreozzi $(2010,2012)$ is the first to investigate the consequences of investment cost heterogeneity. He retains the same basic structure as in Troger (2002) and Ellingsen and Robles (2002) so that, in each period, the size of the pie is determined by the investment decision of one agent only; however, the population of investing agents is now assumed to contain a fraction of efficient agents (those with low investment cost) and a fraction of inefficient agents (those with high investment cost). When the evolved pie share is sufficient to cover the cost of investment for the less efficient agents, then the pie share must also cover the cost of investment for the more efficient ones; when this occurs the resulting equilibrium is efficient. When instead the evolved pie share gives the incentive to invest to the more efficient agents only, the less efficient ones do not invest and the resulting equilibrium is inefficient. By comparing payoff inequality at the efficient equilibrium with payoff inequality at the inefficient equilibrium, Andreozzi (2010) concludes that efficiency calls for greater inequality

In this paper we extend the existing literature in two directions. Firstly, we introduce cost heterogeneity in a two-stage game in which the pie depends on the investment decisions of two agents. Our game is played by two populations of agents, $A$ and $B$, of equal size. We study the case in which agents $B$ are more efficient than agents $A$ in the sense that they bear a smaller investment cost; the 
investment cost is assumed to be identical for agents of the same population.

This situation may arise, for instance, when (for whatever reason) $A$ and $B$ have different productivity: in order to produce the same amount of output, agents $A$ have to work harder and/or for a longer period of time than agents $B .{ }^{3}$

We assume that when both agents invest, they get involved in a joint activity generating a big pie. When instead only one agent invests, an individual activity is undertaken generating a small pie. Lastly, no pie is produced when nobody invests. A neat example of this situation is provided by whale-hunting at Lamalera (Alvard and Nolin, 2002). Whale-hunting is a risky activity which is possible only if all hunters cooperate in the production stage, by going to see on the same boat. This makes whale-hunting a joint venture, an activity impossible to accomplish by a solitary hunter. The alternative is small-game hunting, an individual activity which does not require any cooperation in the production stage. In terms of our model, whale-hunting corresponds to a symmetric investment profile while individual small-game hunting corresponds to an asymmetric investment profile. ${ }^{4}$

Secondly, we consider the case in which the bargaining game played in the second stage is sensitive to the investment profile observed and to the degree of property rights protection. We assume that when both agents invest (so that a big pie is produced), a Nash Demand Game (NDG) occurs; when nobody invests there is no bargaining since there is no pie. What becomes critical is the bargaining occurring when an asymmetric investment profile is observed (so

\footnotetext{
${ }^{3}$ Perhaps some reader might deem more adequate to suppose that agents could choose the partner with whom to interact. In this case group selection may lead the efficient agents to interact with other efficient agents only. This perspective is not considered here.

${ }^{4}$ The situation we have in mind is thus close to the game of life found in primitive economies after the discovery of large-game hunting. These high quality food packages required the cooperation of several hunters and were impossible to acquire by a solitary hunter (Boehm, 2012). Our model is thus not intended to represents the situation in which production is individually pursued even when both agents invest. In this latter case egalitarianism may still evolve; however, our conjecture is that its driving force has now to be found in risk-sharing arrangements. This is the subject of ongoing reseach.
} 
that a small pie is produced). Two different scenarios are considered, depending on whether property rights over the fruits of one's own independent investment are secured or not. When property rights are secured, we explore the case in which the bargaining stage is described by a Dictator Game (DG) in which the unique investing agent has full control of the surplus division. This is intended to describe a situation in which sharing is contingent upon contribution. Those who do not contribute might still receive a share of the small pie, but this is entirely dependent on investor's will. When instead individual property rights are not secured, we explore the case in which the bargaining stage is described by an Ultimatum Game (UG) in which the unique investing agent proposes a division which is realized only if accepted by the opponent. This is intended to describe a situation in which, since the fruits of one's own independent investment are communal (rather than private) property, even those who do not contribute to the pot are entitled to a rigth-to-a-share (Bell, 1985) of the small pie. The division must thus respect this right. This, in turn, means that the unique investing agent is not free to decide how to divide the fruits of her own independent investment; rather she must share - and she must share fairly - to avoid costly dispute. ${ }^{5}$

Unlike previous contributions ${ }^{6}$, we thus study a setup where property rights

\footnotetext{
${ }^{5}$ Among hunter-gatherers, a meat division is not a fictitious game: feeling treated unfairly may easily give rise to violent confrontations (what we called "costly dispute"). According to Boehm (2004), "people in bands feel strongly about their meat-distribution systems and about large game carcasses being essentially communal property (...); they can become quite aggressive (...) if these norms are seriosly violated"

${ }^{6}$ Dawid and MacLeod (2001) is the contribution closer to our model. However, in our opinion their way of shaping the distribution stage is rather critical. Indeed they assume that equal split occurs after a symmetric investment profile while a NDG occurs after an asymmetric investment profile. They defend the equal division assumption by appealing to Young (1993) who, however, proved this result for an exogenously given pie. The equal split assumption is even more critical in our model since the two agents have different investment costs. The other assumption, namely that a NDG occcurs after an asymmetric investment profile, can effectively be adequate in some context; nevertheless we contend its general validity. In a preliminary study we considered the case in which a NDG, rather then an UG, occurs after an asymmetric investment profile; however we were not able to derive the stochastically stable equilibrium due to the occurrence of several non-singleton absorbing sets with complicated structure.
} 
on the fruits of individual investment are strong and compare this with another setup in which those property rights are slightly reallocated by granting a vetoright to a non-investing partner. ${ }^{7}$

We are therefore concerned with two different two-stage games, that we label $\Gamma_{D G}$ and $\Gamma_{U G}$, which only differ in the bargaining rule following an asymmetric investment profile. ${ }^{8}$ Both games have a multiplicity of equilibria. In order to identify the evolved social norms we use the concept of stochastic stability and claim that a social norm exists if the stochastically stable outcome is unique. The existing literature focuses mostly on whether evolution favours efficient norms of distribution; in this paper, instead, we are mostly concerned with the evolution of egalitarianism, i.e. a state of economic affairs minimizing payoff inequality for all members of society. Since in our model agents are risk neutral, payoff are given by the share of the gross surplus received minus the investment cost (if any). Given cost heterogeneity among the two populations, payoff inequality is observed even if the gross surplus is equally distributed. Our main result is that egalitarianism is more likely to emerge in situations in which property rights over the fruits of one's own independent investment are not secured. More precisely, we show that in $\Gamma_{D G}$ the stochastically stable convention is always the one in which the efficient pie is produced and is equally divided. We also show that in $\Gamma_{U G}$, when a unique stochastically stable convention exists, it supports the efficient pie; in this latter case, however, two divisions of the gross surplus may evolve: one supporting equal sharing and one granting a larger

\footnotetext{
${ }^{7}$ Of course, other plausible alternatives could be considered; nevertheless, we believe that our choice has the merit to adequately highlight the role of property rights protection in the evolution of investment and bargaining behavior. We leave to further research to investigate the robustness of our results

${ }^{8}$ Evidence from anthopology suggests that the two games, $\Gamma_{D G}$ and $\Gamma_{U G}$, are indeed plausible. For instance, Hill (2002) reports that sharing conditioned upon contribution is observed among the Ache of Paraguay. On the other hand, Boehm (2004, 2012) says that individuals living in small nomadic bands often do not have the right to treat the carcasses they have killed as private property. This occurs, for instance, among the !Kung and the Hazda, where forms of sharing not contingent upon contribution are observed (Woodburn, 1982).
} 
share to the less efficient agent. ${ }^{9}$

The intuition behind these results is the following. We know from Young (1993) that - provided some homogeneity conditions are satisfied ${ }^{10}$ - the NDG has a natural tendency to evolve towards equal division. In our case, since the pie is endogenous, we need to show that equal sharing provides an adequate incentive to invest. This, in turn, crucially depends on what agents can get by not investing, i.e. the outside option. However, since there is no stable norm regulating the distribution of gross surplus at an asymmetric investment profile, the outside option arises under evolutionary drift of agents' beliefs. ${ }^{11}$ The crucial point is that different rules of the game have different impact on drifting beliefs.

To see this, let's suppose drift lead an agent to believe to appropriate the small pie produced by another investing agent with whom she interacts. Suppose this belief effectively induces the former agent to refrain from investing. In $\Gamma_{D G}$ the initial belief turns out to be a mistake because the single investing agent, behaving as a dictator, gets the entire small pie (or its largest feasible division). Learning this is enough to induce our agent to invest when she believes that the big pie will be equally divided. In $\Gamma_{U G}$ instead the initial belief may not be contradicted by the rules of the game. Two situations are now possible, depending on the size of the efficient pie. Equal sharing evolves when, at the efficient equilibrium, the gross surplus is so large to induce all agents to invest

\footnotetext{
${ }^{9}$ From a static analysis, $\Gamma_{D G}$ and $\Gamma_{U G}$ are essentially identical in the sense that the subgame perfect equilibrium sustaining the efficient investment profile is the same in the two games. However, the evolutionary dynamics are quite different in the two contexts. The fact that evolutionary dynamics of the kind we consider do not necessarily select outcomes resulting from backward induction is known; see, for instance, Noldeke and Samuelson (1993) and Samuelson (1997).

${ }^{10}$ These conditions hold in our case since agents are risk neutral and have the same information (sample size) on opponents' past actions.

${ }^{11}$ As explained in details in Section 3, we adopt the evolutionary framework put forward by Noldeke and Samuelson (1993) in which agents learn opponents' actions played at the node reached by current play only. Beliefs about opponents' actions in nodes not reached by current play are instead subject to drift and can take any value.
} 
even when they believe to obtain the entire small pie produced by the opponent alone (i.e the original belief induced by drift). When instead at the efficient equilibrium the gross surplus is more limited - so that equal sharing no longer gives the incentive to invest to the less efficient agent when she believes to obtain the entire small pie produced by the opponent alone - a new norm of division evolves, giving to the less efficient agents $A$ a larger share.

This can be grasped by looking at Figure 1 , in which $\Gamma_{D G}$ and $\Gamma_{U G}$ are contrasted. In this Figure we assume that when one agent only invests a small pie valued 40 is produced, that the investment cost for the less efficient agent $A$ is 20 and that the more efficient agent $B$ bears no investment cost. The three decreasing lines of dots represent respectively the payoff pairs in the case in which both $A$ and $B$ invest (upper line), only $B$ invests (middle line) and only $A$ invests (lower line). ${ }^{12}$ The upper increasing dotted line represents the payoff pair corresponding to equal division of the gross surplus; the lower increasing dotted line represents the gross surplus divisions generating payoff equality. In each panel, the big dot represents the payoff profile corresponding to the stochastically stable equilibrium. The dotted square represents the set of payoff such that both agents get at least a payoff of 40 (this is the maximum payoff an agent can obtain by not investing; given risk neutrality, it occurs when this agent gets the entire small pie produced by the other). Figure 1 shows that case in which stochastic stability supports the equal division of the big efficient pie in $\Gamma_{D G}$ but not in $\Gamma_{U G}$. In this latter game, in fact, the size of the efficient pie is such that less efficient agent $A$ gets at least 40 (i.e. the maximum one can hope to obtain by not investing) only if the efficient pie is divided unequally; the stochastically stable convention is then the convention closest to the equal division giving to $A$ a payoff equal to $40 .^{13}$

\footnotetext{
${ }^{12}$ To each of these decreasing lines there corresponds a given gross surplus. For any payoff pair resulting from a division of this gross surplus, the sum of the payoff gives the net surplus.

${ }^{13}$ Other two situations, not depicted in Figure 1, can arise in $\Gamma_{U G}$. In one case the big pie
} 
These results have an interesting consequence for the inequality pattern observed in the two games. When the size of the efficient pie is large enough, payoff inequality is the same in the two games; when instead the size of the efficient pie is more limited (i.e. the case illustrated in Figure 1), payoff inequality in $\Gamma_{U G}$ is less than in $\Gamma_{D G}$.

The paper is organized as follows. In Section 2 we present the model. The evolutionary dynamics are studied in Section 3 and the main results are presented in Section 4. Section 5 contains some concluding remarks. All the proofs are in the Appendix.

FIGURE 1 HERE

\section{The model}

Two risk neutral players $(A$ and $B)$ are engaged in a two-stage game. In stage one both have to simultaneously decide whether to invest (action $H$ ) or not (action $L$ ) in producing a pie. Investment costs are $c_{A}$ and $c_{B}$, respectively. When both choose $H$, agents are engaged in a joint activity and the resulting gross surplus is $V_{H}$; when instead only one chooses $H$, an individual activity is realized and the gross surplus is $V_{M}$; lastly, when both choose $L$ there is no surplus. Obviously, $V_{H}>V_{M}>0$. At the end of stage one the produced surplus is observed and each player can correctly estimate her opponent's choice. In stage two they bargain over the available gross surplus. If both invest, they are engaged in a Nash Demand Game (NDG). If only one invests, the paper

is so small that there is no way to divide it such that both players get at least 40 (the upper decreasing line has no intersection with the dotted area). In this case, stochastic stability provides no guide to select one convention in $\Gamma_{U G}$ while equal pie division is still the evolved convention in $\Gamma_{D G}$. In the other case the big pie is is so big that dividing it equally yields both agents a payoff bigger than 40 ; stochastic stability now select the same convention in both games. 
explores two possibilities: either an Ultimatum Game (UG) or a Dictator Game (DG).

We denote by $\Gamma_{U G}\left(\right.$ resp. $\left.\Gamma_{D G}\right)$ the whole extensive game in which a NDG occurs when both players invest and a UG (resp. DG) occurs when only one invests. In NDG players $A$ and $B$ simultaneously make demands $y$ and $x$, respectively. If these demands are compatible, each receives what she claimed; otherwise they receive nothing. Since agents are risk neutral, the payoffs are $\pi_{A}=y-c_{A}$ and $\pi_{B}=x-c_{B}$ if $y+x \leq V_{H} ; \pi_{A}=-c_{A}$ and $\pi_{B}=-c_{B}$ if $y+x>V_{H}$. In UG the player who invests makes a proposal which the opponent can either accept or reject. In DG the division continues to be advanced by the player who invests; however her opponent now has no choice but to accept. Let $D\left(V_{j}\right)=\left\{\delta, 2 \delta, \ldots, V_{j}-\delta\right\}, j \in\{H, M\}$ denote the set of feasible claims.

Throughout the paper we make the following assumption:

Assumption 1 (a) $V_{H} / 2, V_{M}, c_{A}$ and $c_{B}$ are all divisible by $\delta$;

(b) $c_{A} \geq c_{B}>\delta$;

(c) the maximum payoff attainable by playing $H$ when the opponent chooses $L$ is positive, i.e.

$$
c_{A}<V_{M}-\delta
$$

(d) the efficient net surplus arises when both players choose H, i.e.

$$
V_{H}-c_{A}-c_{B}>V_{M}-c_{B}
$$

(e) the population is sufficiently large, i.e.

$$
\frac{V_{H}}{N}<\delta
$$

When $\delta$ is negligible, points (b), (c) and (d) of Assumption 1 are satisfied 
when:

$$
\begin{array}{lll}
c_{B} \leq c_{A}<V_{M} & \text { if } & V_{M} \leq \frac{V_{H}}{2} \\
c_{B} \leq c_{A}<V_{H}-V_{M} & \text { if } & V_{M}>\frac{V_{H}}{2} .
\end{array}
$$

It is worth noticing that, under Assumption 1 , both $\Gamma_{U G}$ and $\Gamma_{D G}$ admit a subgame perfect equilibrium which supports the investment profile $H H$. However, both games admit a great number of inefficient subgame perfect equilibria. As stated in Result 1 below, there is a one-to-one relationship between the subgame-perfect equilibria supporting the full investment profile in the two scenarios considered; this means that, in a static context, the difference between the two scenarios is negligible ${ }^{14}$.

Result 1 For each Nash equilibrium supporting the efficient investment profile in $\Gamma_{D G}$, there exists a Nash equilibrium in $\Gamma_{U G}$ supporting the same investment profile and the same distribution of the gross surplus (and vice versa), provided $c_{B}+\delta<x<V_{H}-c_{A}-\delta$.

Proof. See the Appendix.

\section{$3 \quad$ Evolutionary dynamics}

We embed our model into the evolutionary framework put forward by Noldeke and Samuelson (1993). To this end we postulate a finite population of size $N$ for each player, $A$ and $B$. In each period, every possible match between agents occurs meaning that each agent belonging to population $A$ interacts with each agent of population $B$, one at a time. We assume that investment costs and payoffs are private information. An agent is described by a characteristic which consists of a detailed plan of (pure) actions and a set of beliefs about the

\footnotetext{
${ }^{14}$ We thank an anonymous referee for suggesting this result.
} 
opponent's behavior. ${ }^{15}$

Let $\theta$ be a state; this denotes a profile of characteristics of the overall population; $z(\theta)$ denotes the distribution across the terminal nodes generated by $\theta$; lastly, $\Theta$ is the finite set of possible states.

Evolutionary dynamics are driven by learning and mutation. At the end of every period, with probability $\lambda$ an agent observes the distribution of outcomes $z(\theta)$. This information allows agents to correctly update their beliefs on opponent's choices at the reached information set; given these new beliefs, they also update their action profile by choosing a best reply ${ }^{16}$. Notice, however, that when an agent has the opportunity to learn, she only observes the distribution of outcomes but not the payoff received by the other agents. The following assumption is introduced for the sake of simpler proofs.

Assumption 2 When an agent is indifferent between action $H$ and action $L$ she will choose $L$.

The learning mechanism engenders an unperturbed Markov process $(\Theta, P)$ where $P$ is the transition matrix on $\Theta$. By $\Omega$ we denote a generic absorbing set of the unperturbed process; this is a minimal subset of states such that, once entered, is never abandoned. The basin of attraction of an absorbing set is the set of states $\beta(\Omega)$ such that, for all $\theta \in \beta(\Omega)$, a path from $\theta$ to $\theta^{\prime} \in \Omega$ is observed with positive probability. A state $\theta$ is an equilibrium ${ }^{17}$ of the unperturbed process if no alternative state $\theta^{\prime}$ can be reached from $\theta$. By $\Sigma^{U}$ (resp. $\Sigma^{D}$ ) we

\footnotetext{
${ }^{15}$ In $\Gamma_{U G}$ the plan of actions for player $A$ must specify: (i) the type of investment; (ii) the demand when both players choose $H$; (iii) the demand when $A$ chooses $H$ and $B$ chooses $L$; (iv) whether to accept the demands made by $B$ when, in the first stage, $B$ chooses $H$ and $A$ chooses $L$. In $\Gamma_{D G}$ the plan of actions for player $A$ must specify: (i) the type of investment; (ii) the demand when both players choose $H$; (iii) the division of the surplus when, in the first stage, $A$ chooses $H$ and $B$ chooses $L$. Analogously for player $B$.

${ }^{16}$ When there are multiple best replies, one of them is chosen at random according to a distribution with full support.

${ }^{17}$ In particular in our case a state $\theta$ is an equilibrium state if and only if it is a self-confirming equlibrium of the stage game. See Noldeke and Samuelson (1993) for a definition.
} 
denote the union of absorbing sets of the unperturbed process in $\Gamma_{U G}$ (resp. $\left.\Gamma_{D G}\right)$.

Besides being updated, agents' beliefs and actions can also change by mutation. In every period each agent has a probability $\epsilon$ of mutating. When mutating, agents change their characteristics according to a probability distribution assigning positive probability on each possible characteristic. If this mutation changes the action and/or belief at a decision node that is not currently reached, this mutation is called drift.

Mutations allows the system to switch from an absorbing set to another one. A collection of absorbing sets is a mutation-connected set $X$ if every state can be reached from every other state in the set by a sequence of one-mutation transitions. A mutation-connected set $X$ is locally stable if for any $\Omega \in X$ a single mutation is not enough to enter the basin of attraction of another $\Omega^{\prime} \notin X$.

Mutations generate a new perturbed Markov process $(\Theta, P(\epsilon))$ which is ergodic; hence it has a unique stationary distribution $\mu_{\epsilon}$. Let $\mu_{*}=\lim _{\epsilon \rightarrow 0} \mu_{\epsilon}$ denote the limit distribution. A state $\theta$ is stochastically stable if $\mu_{*}(\theta)>0$ (Foster and Young, 1990). Noldeke and Samuelson (1993) proved that: (i) the stochastically stable set is contained in the union of the absorbing sets of the unperturbed process; (ii) if an absorbing set $\Omega$ is contained in the support of $\mu_{*}$, then all absorbing sets which are reachable from $\Omega$ by a sequence of singlemutation transitions are contained in the support of $\mu_{*}$; (iii) local stability is a necessary condition for stochastic stability. When the set of stochastically stable states contains equilibria supporting the same unique outcome we can speak of a stochastically stable outcome rather than a stochastically stable set.

We denote by $\Sigma_{S}^{\eta}, \eta \in\{U, D\}$, the set of stochastically stable states of $\Gamma_{U G}$ and $\Gamma_{D G}$. From now on, by slightly abusing notation, $\left(H H, y_{H H}, x_{H H}\right)$ denotes the outcome in which both agents have invested, agent $A$ demands $y_{H H}$ and 
agent $B$ demands $x_{H H}$. This also applies to other outcomes. We denote by $\rho(\theta)$ the set of outcomes that can be observed given $\theta$.

Definition 1 Consider the following classes of equilibria:

$$
\begin{aligned}
& \Sigma_{H}^{\eta}=\left\{\theta \mid \rho(\theta)=\left\{\left(H H, V_{H}-x_{H H}, x_{H H}\right)\right\}\right\} \\
& \Sigma_{H L}^{\eta}=\left\{\theta \mid \rho(\theta)=\left\{\left(H L, V_{M}-x_{H L}, x_{H L}\right)\right\}\right\} \\
& \Sigma_{L H}^{\eta}=\left\{\theta \mid \rho(\theta)=\left\{\left(L H, V_{M}-x_{L H}, x_{L H}\right)\right\}\right\} \\
& \Sigma_{L}^{\eta}=\{\theta \mid \rho(\theta)=\{(L L, 0,0)\}\} .
\end{aligned}
$$

We denote by $\theta_{x}$ an equilibrium belonging to $\Sigma_{H}^{\eta}$ with $\left(V_{H}-x, x\right)$. The next two Propositions give the properties of the absorbing sets.

Proposition 1 Consider $\Gamma_{U G}$ and let Assumptions 1 and 2 be satisfied. Then:

(a) all classes of equilibria in Definition 1 are not empty and there is a collection of non-singleton absorbing sets $\Omega_{c}$ such that $\Sigma^{U}=\Omega_{c} \cup \Sigma_{H}^{U} \cup \Sigma_{H L}^{U} \cup$ $\Sigma_{L H}^{U} \cup \Sigma_{L}^{U}$

(b) from any absorbing set, an equilibrium $\theta_{x} \in \Sigma_{H}^{U}$ can be reached by a sequence of single-mutation transitions.

Proof. See the Appendix.

In $\Gamma_{D G}$ it is immediate to observe that in every match with a non-investing agent, every investing agent always demands the largest feasible share of the small pie, $V_{M}-\delta$. Then every equilibrium in $\Sigma_{H L}^{D}\left(\right.$ resp. $\left.\Sigma_{L H}^{D}\right)$ only supports the outcome $\left(V_{M}-\delta, \delta\right)$ (resp. $\left.\left(\delta, V_{M}-\delta\right)\right)$. Moreover, $\Sigma_{L}^{D}$ is empty since the best reply to not investing is always to invest and demand $V_{M}-\delta$.

Proposition 2 Consider $\Gamma_{D G}$ and let Assumptions 1 and 2 be satisfied. Then: (a) $\Sigma^{D}=\Sigma_{H}^{D} \cup \Sigma_{H L}^{D} \cup \Sigma_{L H}^{D}$; 
(b) from any absorbing set, an equilibrium $\theta_{x} \in \Sigma_{H}^{D}$ can be reached by a sequence of single-mutation transitions.

Proof. See the Appendix.

The difference between the two games is that every absorbing set in $\Gamma_{D G}$ is singleton while this is not true for some of the absorbing sets in $\Gamma_{U G}$. The occurrence of a non-singleton absorbing set critically hinges upon agents' beliefs on what happens at asymmetric investment profiles; this explains why it does not occur in models in which the pie is produced by the investment of a single agent. ${ }^{18}$ The intuition is that in $\Gamma_{D G}$ it is never profitable to be the only agent who does not invest since she will get nothing (or the smallest share, $\delta$ ); this fact pushes learning dynamics towards an absorbing set containing a single outcome. In $\Gamma_{U G}$ instead many beliefs on payoff at an asymmetric investment profile can be sustained; for some of them the learning dynamics are trapped in a cycle connecting different outcomes. ${ }^{19}$ This occurs, for instance, when at $\theta$ we have ${ }^{20}$ :

(i) $\rho(\theta)=\left\{\left(H H, \delta, V_{H}-\delta\right) ;\left(H L, V_{M}-\delta, \delta\right) ;\left(L H, V_{M}-c_{B}, c_{B}\right) ;(L L, 0,0)\right\}$;

(ii) all agents $B$ deem that at $L H$ opponents reject a share smaller than $V_{M}-c_{B}$;

(iii) all agents have just updated and $p_{A}(\theta)=p_{B}(\theta)=1 / 2$.

As it can be easily verified, starting from $\theta$, the process can not reach the basin of attraction of an equilibrium belonging to $\left(\Sigma_{H}^{U} \cup \Sigma_{H L}^{U} \cup \Sigma_{L H}^{U} \cup \Sigma_{L}^{U}\right)$ but rather it cyclically visits all the four listed outcomes. Hence $\theta$ belongs to an absorbing set $\Omega$ such that $\rho(\Omega)$ includes all the observed outcomes.

\footnotetext{
${ }^{18}$ A non-singleton absorbing set exists also in Dawid and MacLeod (2001).

${ }^{19}$ As a consequence of this fact, the process does not satisfy the no-cycling condition; see Binmore, Samuelson and Young (2003).

${ }^{20}$ The interested reader can verify that the example corresponds to the case studied in the proof of Proposition 1 in which $A_{1}<0, A_{2}>0, B_{1}>0$ and $B_{2}=0$.
} 


\section{Main results}

In this Section we show that a unique stochastically stable outcome always exists in $\Gamma_{D G}$ and it supports the efficient investment profile and the equal surplus division. We also show that in $\Gamma_{U G}$ a unique stochastically stable outcome only emerges under some circumstances; in this case the long run outcome continues to support the efficient investment profile but surplus division may not be equal. Propositions 3 and 4 are the main results of the paper.

We start from $\Gamma_{D G}$. Under Assumption 2 the equilibria in $\Sigma_{H}^{D}$ support a pair of compatible demands $\left(V_{H}-x, x\right)$ such that:

$$
c_{B}+2 \delta \leq x \leq V_{H}-c_{A}-2 \delta .
$$

For $\delta$ sufficiently small the set $\Sigma_{H}^{D}$ is well defined and it contains the equilibrium $\theta_{x}$ in which the surplus is equally shared.

Consider now $\Gamma_{U G}$. Let $x_{B}^{U}$ (resp. $V_{H}-x_{A}^{U}$ ) denote the share of the large pie going to player $B$ (resp. $A$ ) such that she receives an equilibrium payoff equal to the largest feasible share of the small pie, $V_{M}-\delta$ :

$$
\begin{array}{ll}
x_{B}^{U}-c_{B} & =V_{M}-\delta \\
V_{H}-x_{A}^{U}-c_{A} & =V_{M}-\delta .
\end{array}
$$

Hence, when $B$ believes to get $V_{M}-\delta$ in $H L$, she is induced to invest provided she receives at least $x_{B}^{U}+\delta$. Analogously, when $A$ believes to get $V_{M}-\delta$ in $L H$, she is induced to invest provided she receives at least $V_{H}-\left(x_{A}^{U}-\delta\right)$. We have $x_{B}^{U}+\delta \leq x_{A}^{U}-\delta$ if:

$$
c_{A}+c_{B} \leq V_{H}-2 V_{M} .
$$


When this condition is satisfied, we can define the following set

$$
\Sigma_{I H}^{U}=\left\{\theta_{x} \mid x \in\left[x_{B}^{U}+\delta, x_{A}^{U}-\delta\right]\right\}
$$

which represents the set of equilibria in which both agents invest and $x \in$ $\left[x_{B}^{U}+\delta, x_{A}^{U}-\delta\right]$. The set $\Sigma_{H}^{U}$ can thus be partitioned into $\Sigma_{I H}^{U}$ and $\Sigma_{C H}^{U}=$ $\Sigma_{H}^{U} \backslash \Sigma_{I H}^{U}$ where $\Sigma_{C H}^{U}$ denotes the set of equilibria in which both agents invest but $x \notin\left[x_{B}^{U}+\delta, x_{A}^{U}-\delta\right]$. Hence, $\Sigma_{H}^{U}=\Sigma_{I H}^{U} \cup \Sigma_{C H}^{U}$ when (7) holds and $\Sigma_{H}^{U}=\Sigma_{C H}^{U}$ when (7) doesn't hold.

By definition, at any $\theta_{x} \in \Sigma_{I H}^{U}$ each agent receives an equilibrium payoff not smaller than the maximum payoff attainable when she deviates by playing $L$; any equilibrium in $\Sigma_{I H}^{U}$ thus dominates all the equilibria supporting other investment profiles. Hence, even if at an equilibrium $\theta_{x} \in \Sigma_{I H}^{U}$ the beliefs on the $H L$ outcome drift, allowing some agents to expect to get almost the whole surplus if they do not invest, this drift does not push the process away from the basin of attraction of $\theta_{x}$. This is not true for any $\theta_{x} \in \Sigma_{C H}^{U}$.

Condition (7) is compatible with point (b) of Assumption 1 provided $\frac{V_{H}}{2}-$ $c_{B} \geq V_{M}$ saying that, for the more efficient agent $B$, investing and getting half of the bigger pie is better than not investing and getting the entire smaller pie. However, this last condition is compatible with point (c) of Assumption 1 only for $c_{B}<\frac{V_{H}}{4}$. Hence, under Assumption 1, condition (7) is never satisfied when $c_{B} \geq \frac{V_{H}}{4}$. The following Proposition summarizes our result concerning the evolution of an investment norm in both games.

Proposition 3 Let $\delta$ be sufficiently small and let Assumptions 1 and 2 be satisfied. Then:

(a) the equilibria $\theta_{x} \in \Sigma_{H}^{D}$ are locally stable;

(b) when (7) holds, the equilibria $\theta_{x} \in \Sigma_{I H}^{U}$ are locally stable while the equilibria $\theta_{x} \in \Sigma_{C H}^{U}$ are not; 
(c) when (7) doesn't hold, the set $\Sigma_{C H}^{U} \cup \Sigma_{L}^{U} \cup \Sigma_{L H}^{U} \cup \Sigma_{H L}^{U}$ is mutation connected and locally stable.

Proof. See the Appendix

Point (a) states that evolution always selects the efficient equilibrium in $\Gamma_{D G}$; this occurs in region $A D E$ of Figure 2. Points (b) and (c) state that not all the efficient equilibria evolve in $\Gamma_{U G}$; rather evolution only selects the equilibria in $\Sigma_{I H}^{U}$. This, of course, is only possible when $\Sigma_{I H}^{U}$ is not empty, condition satisfied when (7) holds; this occurs in region $A C F$ of Figure 2 in which investments are complements and the total investment cost is limited. According to Dawid and MacLeod (2008), investments are complements if the surplus gain resulting from investing when the opponent invests is greater that the surplus gain resulting from investing when the opponent does not invest; in our case this is satisfied if $V_{M}<\frac{1}{2} V_{H}$. When instead condition (7) is violated, $\Sigma_{I H}^{U}$ is empty and $\Sigma_{H}^{U}=\Sigma_{C H}^{U}$; since the set $\Sigma_{C H}^{U} \cup \Sigma_{L}^{U} \cup \Sigma_{L H}^{U} \cup \Sigma_{H L}^{U}$ is mutation connected and locally stable, one mutation is enough to move from one equilibrium to another without leaving the whole set; as a consequence, all the outcomes belonging to this mutation connected set are stochastically stable. All this occurs in region $C D E F$ of Figure 2 which corresponds to the case in which either investments are not complements or investments are complements but total investment cost is too high.

\section{FIGURE 2 HERE}

Before turning our attention to the bargaining norm we observe that, under condition (7), the set $\Sigma_{I H}^{U}$ is compatible with two different settings. In the first we have $x_{B}^{U}+\delta<\frac{V_{H}}{2} \leq x_{A}^{U}-\delta$; this occurs when

$$
c_{A} \leq \frac{V_{H}}{2}-V_{M}
$$


In the second we have instead $x_{B}^{U}+\delta \leq x_{A}^{U}-\delta<\frac{V_{H}}{2}$; this occurs when

$$
\frac{V_{H}}{2}-V_{M}<c_{A} \leq V_{H}-2 V_{M}-c_{B}
$$

In the next Proposition we derive the stochastically stable outcome for each game. Stochastic stability basically calls for counting the number of mutations needed to move from one equilibrium to another. Since $\Gamma_{D G}$ satisfies the nocycling condition, we can use the naive minimization test (Binmore et al. 2003). However this test can not be used in $\Gamma_{U G}$ since this game does not satisfy the no-cycling condition (see point (a) of Proposition 1); in this case we have no alternative but to resort to the sufficient condition developed in Ellison (2000) and known as radius modified coradius criterion. Both techniques are briefly summarized in the Appendix.

Proposition 4 Let $\delta$ be sufficiently small and let Assumptions 1 and 2 be satisfied. Then:

(a) $\Gamma_{D G}$ has always a unique stochastically stable outcome; this supports the efficient equilibrium and the equal surplus division, i.e. $\left(V_{H} / 2, V_{H} / 2\right)$;

(b) $\Gamma_{U G}$ has a unique stochastically stable outcome provided condition (7) holds; this supports the efficient equilibrium and a surplus division which is either $\left(V_{H} / 2, V_{H} / 2\right)$ if $c_{A} \leq \frac{V_{H}}{2}-V_{M}$ or $\left(V_{M}+c_{A}, V_{H}-V_{M}-c_{A}\right)$ if $\frac{V_{H}}{2}-V_{M} \leq$ $c_{A} \leq V_{H}-2 V_{M}-c_{B}$.

Proof. See the Appendix

The intuition of this Proposition is the following. Consider $\Gamma_{D G}$ first. At the efficient equilibrium, the equal split grants to $A$ a payoff $\frac{1}{2} V_{H}-c_{A}$. Suppose $A$ believes to get $V_{M}-\delta>\frac{1}{2} V_{H}-c_{A}$ if she deviates by playing $L$. However, if $A$ 
deviates and $L H$ is observed, then $B$ is a dictator meaning that $A$ 's belief soon turns out to be a mistake since $B$ will only offer $\delta$. By realizing that, $A$ is better off by investing since $\frac{1}{2} V_{H}-c_{A}>\delta$. Consider now $\Gamma_{U G}$. In this game the equal split evolves when $c_{A}$ is limited; this occurs in region $A B F$ of Figure 2. In this case, even if the beliefs of the less efficient agents drift, they can not destabilize the efficient equilibrium being $\frac{1}{2} V_{H}-c_{A}>V_{M}-\delta$. As a result $A$ does not have any incentive to choose $L$. When instead the investment cost of the less efficient agent is bigger, the equal surplus division might not be enough to prevent $A$ from deviating from the efficient equilibrium. This happens if beliefs drift such that $A$ expects to get at $L H$ a payoff $V_{M}-\delta>\frac{1}{2} V_{H}-c_{A}$. In order to induce $A$ to invest, now $B$ must give to $A$ a larger share of the surplus (i.e. $V_{M}+c_{A}$ ) so that $A$ 's payoff is equal to $V_{M}$, slightly bigger than the maximum payoff she could hope to get at $L H$. This occurs in region $B C F$ of Figure 2.

Proposition 4 has an interesting implication for inequality. In both our games, at the observed convention the more efficient agent receives a bigger payoff. In $\Gamma_{D G}$ inequality follows from the same mechanism described by Andreozzi (2012): since all the investing agents receive the same share of the gross surplus, the more efficient agent gets a higher payoff. The same mechanism is at work in $\Gamma_{U G}$ when $c_{A}$ is limited. When instead $c_{A}$ is greater, the mechanism which is responsible for the observed inequality is different; now, in order to provide to the less efficient $A$ the incentive to invest, the evolved bargaining norm has to give to this agent a share of the gross surplus that is larger than the share going to $B$ (still preserving for the latter the incentive to invest). As a consequence, although $\pi_{A}<\pi_{B}$, the observed payoff inequality is now smaller. Since the inequality observed in this last case represents the maximal inequality compatible with cooperation in the production stage, it has a Rawlsian taste. ${ }^{21}$

\footnotetext{
${ }^{21}$ A somehow similar result has been derived by Barling and von Siemens (2010) in the framework of incentivating contracts. They show that equal sharing is the optimal solution to the incentive problems in partnership provided that partners are inequity averse. This
} 


\section{Concluding remarks}

In this paper we extended previous literature on the evolution of social norms in models in which the parties bargain over a pie the size of which is determined by their previous investment decisions. We considered the case in which the pie is determined by the investment decisions of two agents, with different investment costs. Andreozzi $(2010,2012)$ was the first to study the consequence of investment cost heterogeneity. He concluded that efficiency calls for greater inequality. However, Andreozzi (2010) also suggested that his result does not necessarily lend support to the conclusion that, in economies with production, social justice has to be less egalitarian. The main result of the present paper supports this conjecture.

\section{Appendix}

Before giving the proofs we introduce some notation and some preliminary results. Consider a state $\theta$ and suppose that all agents observe $z(\theta)$. In $\Gamma_{U G}$ an agent $i \in A$ chooses $H$ if

$$
p_{B}(\theta)\left(\widetilde{y}_{H H}^{i}(\theta)-\widetilde{y}_{L H}^{i}(\theta)-c_{A}\right)+\left(1-p_{B}(\theta)\right)\left(\widetilde{y}_{H L}^{i}(\theta)-c_{A}\right)>0 ;
$$

an agent $i \in B$ chooses $H$ if

$$
p_{A}(\theta)\left(\widetilde{x}_{H H}^{i}(\theta)-\widetilde{x}_{H L}^{i}(\theta)-c_{B}\right)+\left(1-p_{A}(\theta)\right)\left(\widetilde{x}_{L H}^{i}(\theta)-c_{B}\right)>0 .
$$

Here we denote by $p_{A}(\theta)$ (resp. $p_{B}(\theta)$ ) the frequency of agents $A$ (resp. $B$ ) who played $H$ in $\theta$, and by $\widetilde{y}_{H H}^{i}(\theta)$ (resp. $\left.\widetilde{x}_{H H}^{i}(\theta)\right)$ the expected payoff of agent

because equal sharing maximizes the incentive of the partner who has the weakest incentive to exert effort. We do not consider inequity aversion. Moreover, in $\Gamma_{D G}$ equal sharing always provides adequate incentive to invest to the less efficient agent. However, in $\Gamma_{U G}$ it provides incentive to invest in region $A B F$ of Figure 4 , but not in region $B C F$. 
$i \in A$ (resp. $i \in B$ ) at the information set $H H$, given $z(\theta)$. Similar conditions hold for $\Gamma_{D G}$. Following Young (1993) we denote by $\lceil s\rceil$ the least integer number greater than $s$ when $s$ is not an integer and $s+1$ otherwise.

Proof of Result 1. In order to prove the statement we show that $\Gamma_{D G}$ and $\Gamma_{U G}$ admit the same subgame perfect equilibrium supporting the efficient investment profile. This implies that, at this equilibrium, the gross surplus is the same in the two cases. Notice first that efficiency requires pie exhaustion when both invest $\left(y_{H H}+x_{H H}=V_{H}\right)$ and when only one invests $\left(y_{H L}+x_{H L}=V_{M}\right.$ at node $H L$ and $y_{L H}+x_{L H}=V_{M}$ at node $\left.L H\right)$. Now observe that $A$ invests if $y_{H H}-c_{A}>y_{L H}$ while $B$ invests if $x_{H H}-c_{B}>x_{H L}$. Consider $\Gamma_{U G}$ first. The maximal punishment $A$ can impose upon $B$ for not investing is to demand $y_{H L}^{P}=V_{M}-\delta$ for herself so that $x_{H L}^{P}=\delta$. Agent $B$ will play $H$ if $x_{H H}-c_{B}>$ $x_{H L}^{P}=\delta$. Analogously the maximal punishment $B$ can impose upon $A$ for not investing is to demand $x_{L H}^{P}=V_{M}-\delta$ for herself so that $y_{L H}^{P}=\delta$. Agent $A$ will play $H$ if $V_{H}-x_{H H}-c_{A}>y_{L H}^{P}=\delta$. Consider now $\Gamma_{D G}$. It is immediate to observe that, given the rules of the game, $y_{H L}=V_{M}-\delta\left(\right.$ resp. $\left.x_{L H}=V_{M}-\delta\right)$ is $A$ 's best demand at node $H L$ (resp. $B$ 's best demand at node $L H$ ). It then follows that the same condition we have just derived for $\Gamma_{U G}$ ensures that $\mathrm{HH}$ is subgame perfect equilibrium in $\Gamma_{D G}$ as well.

Lemma 1 Let $x_{H H, 1}<x_{H H, 2}<\ldots<x_{H H, k}$ be the demands made by $B$ at $\mathrm{HH}$ for some state $\theta$. Then the set of best behavioral demands following $\mathrm{HH}$ for agents $A$ is a subset of $\left\{V_{H}-x_{H H, l}\right\}_{l=1}^{k}$.

Proof. See Lemma A.1 in Ellingsen and Robles (2002)).

Lemma 2 Consider $\Gamma_{U G}$ and let $\Omega$ be a absorbing set. If $\left(H L, y_{H L}, x_{H L}\right) \in$ $\rho(\Omega)\left[\right.$ resp. $\left.\left(L H, y_{L H}, x_{L H}\right) \in \rho(\Omega)\right]$ then: 
(i) $x_{H L}=V_{M}-y_{H L}\left[\right.$ resp. $\left.y_{L H}=V_{M}-x_{L H}\right]$;

(ii) $\left(H L, y_{H L}, x_{H L}\right)\left[r e s p .\left(L H, y_{L H}, x_{L H}\right)\right]$ is the only outcome supporting the investment profile $H L[$ resp. $L H]$.

Proof. We only consider profile $H L$; the same holds true for $L H$.

Point (i). Consider a state $\theta$ belonging to an absorbing set and suppose that $\left(H L, y_{H L}, x_{H L}\right)$ belongs to the support of $z(\theta)$ but $x_{H L} \neq V_{M}-y_{H L}$. Let's suppose that only agents $B$ update their characteristics: they accept $y_{H L}$. Denote by $\theta^{\prime}$ this new state reachable from $\theta$. Since $\theta$ is not accessible from $\theta^{\prime}$, then $\theta$ can not belong to an absorbing set.

Point (ii). Consider a state $\theta$ belonging to an absorbing set and suppose that multiple demands are made by agents $A$ at $H L$. We already know from point (i) that agents $B$ accept the demands made by their opponents. Suppose only agents $A$ revise their characteristics. In the newly reached state $\theta^{\prime}$, any agent $A$ will make the maximum demand $\left(y^{*}\right)$ observed at $H L$ and no agent will have the incentive to deviate from the action profile $\left(y^{*}, V_{M}-y^{*}\right)$. Since $\theta$ is not accessible from $\theta^{\prime}$, then $\theta$ can not belong to an absorbing set. Analogously two states $\theta$ and $\theta^{\prime}$ sustaining a different equilibrium action profile at $H L$ can not belong to the same absorbing state $\Omega$ since these states do not communicate.

Thus, for any absorbing set $\Omega$, only one outcome supporting the $H L$ profile and such that the claims exhaust the surplus can belong to $\rho(\Omega)$.

Lemma 3 Consider either $\Gamma_{U G}$ or $\Gamma_{D G}$ and let $\Omega$ be a absorbing set. If

$\left(H H, y_{H H}, x_{H H}\right) \in \rho(\Omega)$ then:

(i) $x_{H H}=V_{H}-y_{H H}$;

(ii) $\left(H H, y_{H H}, x_{H H}\right)$ is the only outcome supporting the investment profile $H H$.

Proof. Consider a state $\theta$ belonging to an absorbing set $\Omega$ and suppose 
that at least two agents $B$ (resp. $A$ ) have invested but have proposed different demands. Suppose all agents $A$ (resp. $B$ ) revise. With positive probability the process moves from $\theta$ to a new state $\theta^{\prime}$ in which all $A s$ (resp. $B s$ ) choose the same best reply $y^{*}\left(\right.$ resp. $\left.x^{*}\right)$ at $H H$.

Denote by $\widehat{\theta}$ the state accessible from $\theta^{\prime}$ and such that the profile $H H$ occurs for the first time ${ }^{22}$. Consider $\widehat{\theta}$ and let all agents $B$ (resp. $A$ ) update; by Lemma 1 they will demand $V_{H}-y^{*}\left(\right.$ resp. $\left.V_{H}-x^{*}\right)$ at $H H$. In the newly reached state no agent has the incentive to deviate from $\left(y^{*}, V_{H}-y^{*}\right)$ by updating. Therefore states $\theta$ and $\theta^{\prime}$ do not communicate. Then $\theta$ can not belong to $\Omega$. Analogously, two states which sustain a different pair of compatible demands at $H H$ can not belong to the same absorbing set $\Omega$ since these states do not communicate.

Thus, for any absorbing set $\Omega$, only one outcome supporting the $H H$ profile and such that the claims exhaust the surplus can belong to $\rho(\Omega)$.

Proof of Proposition 1. Consider $\Gamma_{U G}$ and let Assumptions 1 and 2 be satisfied. We know from Lemmas 2 and 3 that in any absorbing state only one division is observed. Clearly, all classes of equilibria are not empty.

Point (a). We explore the occurrence of non-singleton absorbing set. We first show that when $\Omega$ is non-singleton, then $\rho(\Omega)$ must include four outcomes (i.e. all possible investment profiles are observed), each of which is an equilibrium. Then we show that from a state $\theta$ belonging to this non-singleton absorbing set it is possible to reach either the basin of attraction of one equilibrium or a state in which all bargaining nodes are observed.

Let $\rho(\Omega)=\left\{\left(H H, V_{H}-x, x\right),\left(H L, V_{M}-x^{*}, x^{*}\right)\right\}$. In $\Omega$ a state $\theta$ supporting both outcomes must exist. Suppose $x-c_{B}>x^{*}$. When $B$ update, they will choose $H$. When $A$ revise, the process moves to $\theta_{x} \in \Sigma_{H}^{U}$ when $\widetilde{y}_{L H}^{i}(\theta)<V_{H}-x-c_{A}$ for all $i \in A$. When instead $\widetilde{y}_{L H}^{i}(\theta) \geq V_{H}-x-c_{A}$

${ }^{22}$ Notice that if $\widehat{\theta}$ does not exist, then $\theta \notin \Omega$ because state $\theta$ is not reachable from $\theta^{\prime}$. 
for some agent $i \in A$, these specific agents choose $L$ so that the state $\theta \in \Sigma_{L H}^{U}$ can be reached. Suppose now $x-c_{B} \leq x^{*}$. When all $B$ update, they choose $L$. When $A$ revise, the process moves either to $\theta \in \Sigma_{L}^{U}$ or to $\theta \in \Sigma_{H L}^{U}$, depending on the sign of $V_{M}-x^{*}-c_{A}$. These facts contradict the assumption that $\rho(\Omega)=\left\{\left(H H, V_{H}-x, x\right),\left(H L, V_{M}-x^{*}, x^{*}\right)\right\}$. It is easy to verify that the same conclusion holds when $\rho(\Omega)$ includes any two different outcomes (of course $\left.\rho(\Omega) \neq\left\{\left(H H, V_{H}-x, x\right),(L L, 0,0)\right\}\right)$. Therefore, when $\Omega$ is non-singleton, $\rho(\Omega)$ includes four outcomes, each of which is an equilibrium.

Let thus $\theta \in \Omega$ be a state in which all the investment profiles are observed. Of course, $0<p_{A}(\theta)<1$ and $0<p_{B}(\theta)<1$. Agents $A$ will choose $H$ if (10) is satisfied while agents $B$ will choose $H$ if (11) holds true; conditions (10) and (11) can be written more compactly as

$$
\begin{aligned}
& p_{B}(\theta) A_{1}+\left(1-p_{B}(\theta)\right) A_{2}>0 \\
& p_{A}(\theta) B_{1}+\left(1-p_{A}(\theta)\right) B_{2}>0
\end{aligned}
$$

where the terms $A_{j}$ and $B_{j}$ have an obvious meaning.

Suppose $A_{1}>0$ and $A_{2} \geq 0$. When updating, agents $B$ play $H$ if $B_{1}>0$ so that the process reaches an state $\theta^{*}$ such that $\rho\left(\theta^{*}\right)=\left\{\left(H H, y_{H H}, x_{H H}\right)\right\}$. Suppose instead $B_{1}>0$ and $B_{2} \geq 0$. When updating, agents $A$ play $H$ if $A_{1}>0$ and the process reaches a state $\theta^{*}$ such that $\rho\left(\theta^{*}\right)=\left\{\left(H H, y_{H H}, x_{H H}\right)\right\}$. By a similar argument we can see that an equilibrium $\rho\left(\theta^{*}\right)=\left\{\left(H L, y_{H L}, x_{H L}\right)\right\}$ is observed when either $A_{1} \geq 0, A_{2}>0$ and $B_{1} \leq 0$ or when $B_{1} \leq 0, B_{2} \leq 0$ and $A_{2}>0$. Moreover, an equilibrium $\rho\left(\theta^{*}\right)=\left\{\left(L H, y_{L H}, x_{L H}\right)\right\}$ is observed when either $A_{1} \leq 0, A_{2} \leq 0$ and $B_{2}>0$ or when $B_{1} \geq 0, B_{2}>0$ and $A_{1} \leq 0$. Lastly an equilibrium $\rho\left(\theta^{*}\right)=\{(L L, 0,0)\}$ is observed when either $A_{1} \leq 0, A_{2} \leq 0$ and $B_{2} \leq 0$ or when $B_{1} \leq 0, B_{2} \leq 0$ and $A_{2} \leq 0$. 
When $B_{1}>0$ (resp. $<0$ ) and $B_{2}<0$ (resp. $>0$ ), agents $B$ play $H$ if $p_{A}(\theta)>p_{A}^{*}\left(\right.$ resp. $\left.p_{A}(\theta)<p_{A}^{*}\right)$ where $p_{A}^{*}=\frac{c_{B}-x_{L H}}{x_{H H}-x_{H L}-x_{L H}}$. When instead $A_{1}>0$ (resp. $A_{1}<0$ ) and $A_{2}<0$ (resp. $A_{2}>0$ ), agents $A$ play $H$ if $p_{B}(\theta)>p_{B}^{*}\left(\right.$ resp. $\left.p_{B}(\theta)<p_{B}^{*}\right)$ where $p_{B}^{*}=\frac{c_{A}-y_{H L}}{y_{H H}-y_{L H}-y_{H L}}$. In these last two cases the process reaches a state $\theta^{*}$ such that either $\rho\left(\theta^{*}\right)=\left\{\left(H H, y_{H H}, x_{H H}\right)\right\}$ or $\rho\left(\theta^{*}\right)=\{(L L, 0,0)\}$ provided that $A_{1} A_{2}<0, B_{1} B_{2}<0$ and $A_{1} B_{1}>0$ and provided one population revises at a time.

The remaining possible cases are those in which either $A_{1}>0, A_{2} \leq 0$, $B_{1}<0$ and $B_{2}>0$ or $A_{1} \leq 0, A_{2}>0, B_{1}>0$ and $B_{2} \leq 0$. In all these cases $\theta \in \beta(\Omega)$ where $\Omega$ is an absorbing set such that $\rho(\Omega)$ includes all the outcomes observed at $\theta$. Denote by $\Omega_{c}$ the collection of all these non-singleton absorbing sets. We conclude that $\Sigma^{U}=\Omega_{c} \cup \Sigma_{H}^{U} \cup \Sigma_{H L}^{U} \cup \Sigma_{L H}^{U} \cup \Sigma_{L}^{U}$.

Point (b). Consider first the case in which the absorbing state is nonsingleton, i.e. $\Omega \in \Omega_{c}$. We already know that at $\Omega$ : (i) the process will cyclically visit all the four investment profiles; (ii) at any bargaining node the demands are compatible; (iii) the claim configuration is such that either $A_{1}>0$, $A_{2} \leq 0, B_{1}<0, B_{2}>0$ or $A_{1} \leq 0, A_{2}>0, B_{1}>0, B_{2} \leq 0$.

Consider a state $\theta \in \Omega$ and suppose $x_{L H}-c_{B} \leq 0$ (i.e. $B_{2} \leq 0$ ); then, necessarily, we must have $A_{1} \leq 0, A_{2}>0$ and $B_{1}>0$. Point (c) of Assumption 1 assures that the outcome $\left(L H, \delta, V_{M}-\delta\right)$ will never be observed at $\theta$. A drift can thus lead all agents $A$ to accept the opponent's maximum feasible demand at $L H$. Suppose now a single agent $B$ mutates her demand from $x_{L H}$ to $V_{M}-\delta$. When all agents $B$ revise, they will demand $V_{M}-\delta$ and play $H$ (since $B_{1}>0$ and $\left.V_{M}-\delta-c_{B}>0\right)$. When all agents $A$ update, their best reply depends on the sign of $y_{H H}-c_{A}-\delta$; however, since $y_{H H}-c_{A}-\delta<0$ when $A_{2}>0$ and $B_{1}>0$, agents $A^{\prime} s$ best reply is $L$. Therefore, by a sequence of single-mutation transitions the process reaches an equilibrium $\theta \in \Sigma_{L H}^{U}$ supporting the outcome 
$\left(L H, \delta, V_{M}-\delta\right)$. A similar argument can be used to show that, when $y_{H L}-c_{A} \leq$ 0 (i.e. $A_{2} \leq 0$ ), by a sequence of single-mutation transitions the process reaches an equilibrium $\theta \in \Sigma_{H L}^{U}$ supporting the outcome $\left(H L, V_{M}-\delta, \delta\right)$.

We are left with the case in which the absorbing state is singleton. The relevant cases are $\Sigma_{H L}^{U} \cup \Sigma_{L H}^{U} \cup \Sigma_{L}^{U}$.

Consider first an equilibrium $\theta \in \Sigma_{L}^{U}$. Let agents' beliefs drift such that: (i) all agents $A$ accept any proposal made by agents $B$ at $L H$; (ii) all agents $A$ expect to get $V_{H}-x>c_{A}+\delta$ at $H H$; (iii) all agents $B$ expect to get the minimum payoff at $H L$; (iv) all agents $B$ expect to get $x>c_{B}+\delta$ at $H H$. Suppose now an agent $B$ mutates by playing $H$ and demanding $V_{M}-\delta$. When agents $B$ update they will choose $H$ since all agents $A$ have accepted. When agents $A$ revise they will play $H$ since $V_{H}-x>c_{A}+\delta$. Hence the process reaches a new equilibrium $\theta_{x} \in \Sigma_{H}^{U}$.

Consider now an equilibrium in which only one agent invests. Let $\theta \in \Sigma_{H L}^{U}$ be such equilibrium and suppose that at this equilibrium a pair of demands $\left(V_{M}-x^{*}, x^{*}\right)$ is observed, with $x^{*}>\delta$. Let agents' beliefs drift such that (i) all agents $B$ accept the maximum feasible demand made by $A$ at $H L$ and (ii) all agents $B$ deem that at $H H$ all agents $A$ shall demand a share larger than $V_{H}-c_{B}+\delta$. Suppose now a single agent $A$ demands $V_{M}-\delta$. Since all $B$ accept this demand, when agents $A$ update they all imitate. When agents $B$ revise they continue to play $L$. Hence, the process reaches a new equilibrium $\theta^{\prime} \in \Sigma_{H L}^{U}$ where $\rho\left(\theta^{\prime}\right)=\left(H L, V_{M}-\delta, \delta\right)$. Consider now this equilibrium $\theta^{\prime}$ and let agents' beliefs drift such that: (i) all agents $A$ expect to get the minimum payoff at $L H$; (ii) all agents $A$ expect to receive $V_{H}-x$ such that $c_{A}+\delta<V_{H}-x<V_{H}-c_{B}-\delta$. Suppose an agent $B$ mutates by playing $H$ and making a demand $x$. When all agents $B$ revise, they will choose $H$ and demand $x$. When agents $A$ update, the process reaches a new equilibrium $\theta_{x} \in \Sigma_{H}^{U}$. A similar argument can be used to 
show that from $\theta \in \Sigma_{L H}^{U}$ it is possible to reach an equilibrium $\theta_{x}$.

Proof of Proposition 2. Consider $\Gamma_{D G}$ and let Assumptions 1 and 2 be satisfied.

Point (a). A non-singleton absorbing set like that described at point (a) in the proof of Proposition 1 cannot exist in $\Gamma_{D G}$. Suppose the contrary and let $\theta$ be a state such that $\rho(\theta)$ includes three outcomes (as described in the main text, $(L L, 0,0)$ cannot be observed). Since, at any absorbing set, $y_{H L}=x_{L H}=V_{M}-\delta$ and $y_{H L}-c_{A}>0$ and $x_{L H}-c_{B}>0$, it follows that the process reaches a state in which one outcome only is observed. This contradicts the initial assumption.

Point (b). Consider an equilibrium $\theta \in \Sigma_{H L}^{D}$ and let the beliefs of agents $A$ drift such that $A$ expect to get a share $\delta+c_{A}<y \leq V_{H}-V_{M}$. Suppose an agent $B$ mutates by playing $H$ and demanding $V_{H}-y$ at $H H$. When all agents $B$ revise they imitate the mutant. When agents $A$ update, the process reaches a new equilibrium $\theta_{x}$.

We know from Propositions 1 and 2 that an efficient equilibrium can be reached by a sequence of single-mutation transition. In order to establish the local stability of this equilibrium we have to know the minimum number of mutations needed to escape, in all possible directions, its basin of attraction. This information is provided by the following three Lemmas. In particular, Lemma 4 gives (for both games) the minimum number of mutations required to leave the basin of attraction of an efficient equilibrium and reaching the basin of attraction of another efficient equilibrium with a different distributional rule. Lemmas 5 and 6 give (for $\Gamma_{D G}$ and $\Gamma_{U G}$ respectively) the minimum number of mutations required to leave the basin of attraction of an efficient equilibrium and reach the basin of attraction of another equilibrium which does not support the efficient investment profile. Let $\widehat{x} \equiv \frac{1}{2} V_{H}$ and denote by $\theta_{\widehat{x}}$ the corresponding equilibrium outcome. 
Lemma 4 Consider $\Gamma_{D G}$ and $\Gamma_{U G}$. The minimum number of mutations needed to get from $\theta_{x}$ (where either $\theta_{x} \in \Sigma_{H}^{D}$ or $\theta_{x} \in \Sigma_{I H}^{U}$ ) to an equilibrium with the same investment profile but different demands is:

$$
\begin{aligned}
& r_{B}^{+}(x)=\left\lceil N\left(\frac{\delta}{V_{H}-x}\right)\right\rceil \text { if } \quad x<\widehat{x} \\
& r_{A}^{-}(x)=\left\lceil N\left(\frac{\delta}{x}\right)\right\rceil \quad \text { if } \quad x>\widehat{x}
\end{aligned}
$$

where $r_{B}^{+}(x)$ is the number of mutations in the $B$ population needed for the transition from $\theta_{x}$ to $\theta_{x+\delta}$ and $r_{A}^{-}(x)$ is the number of mutations in the $A$ population needed for the transition from $\theta_{x}$ to $\theta_{x-\delta}$.

Proof: By a direct application of Young (1993).

Lemma 5 Consider $\Gamma_{D G}$. The minimum number of mutations required to get from $\theta_{x} \in \Sigma_{H}^{D}$ to an equilibrium which supports an inefficient investment profile are:

$$
\begin{aligned}
& \bar{r}_{A}(x)=\left\lceil N\left(1-\frac{\delta+c_{B}}{x}\right)\right\rceil \quad \text { if } \quad x<\widehat{x} \\
& \bar{r}_{B}(x)=\left\lceil N\left(1-\frac{\delta+c_{A}}{V_{H}-x}\right)\right\rceil \text { if } \quad x>\widehat{x} .
\end{aligned}
$$

Proof. We give the proof for $\bar{r}_{B}(x)$ only. We first show that agents are not induced to play $L$ when, in $\theta_{x}$, some opponents mutate and play $L$; then we show that agents are instead induced to play $L$ when some opponents mutate by demanding a larger share.

Consider $\theta_{x} \in \Sigma_{H}^{D}$ and suppose that drift leads agents $A$ to believe to get the maximum payoff in an $L H$ match. Suppose $p_{1}$ agents $B$ mutate by playing $L$ and that this induce all agents $A$ play $L$. A new state $\theta^{\prime} \notin \Sigma_{H}^{D}$ is thus reached. In any $L H$ match, non mutants $B$ behave as dictators and demand $x_{L H}=V_{M}-\delta$. Since all agents $A$ interact with these non mutants $B$, it follows 
that all $A$ learn $y_{L H}=\delta$. After updating their beliefs, agents $A$ play $H$ since $\left(N-p_{1}\right)\left(V_{H}-x-c_{A}-\delta\right)+p_{1}\left(V_{M}-\delta-c_{A}\right)>0$. A new state $\theta^{\prime \prime}$ is thus reached in which all agents $A$ play $H$. When mutants $B$ update, they play $H$ so that a new state $\theta_{x}^{\prime} \in \Sigma_{H}^{D}$ is reached.

Consider again $\theta_{x} \in \Sigma_{H}^{D}$ and suppose $p_{2}$ agents $B$ mutate by claiming $x^{\prime}>$ $x_{A}^{D} \equiv V_{H}-\delta-c_{A}$. Consider the NDG in which agents $B$ either play $x$ or $x^{\prime}>x$ and agents $A$ either play $V_{H}-x$ or $V_{H}-x^{\prime}<V_{H}-x$. A's expected payoff are respectively $\left(V_{H}-x\right) \frac{N-p_{2}}{N}-c_{A}$ and $V_{H}-x^{\prime}-c_{A}$. Hence, $A$ 's best reply to $B$ 's mutations is $V_{H}-x$ if $p_{2} \leq N \frac{x^{\prime}-x}{V_{H}-x}$ and it is $V_{H}-x^{\prime}$ otherwise. When $p_{2} \leq N \frac{x^{\prime}-x}{V_{H}-x}$, agents $A$ play $L$ if $\frac{N-p_{2}}{N}\left(V_{H}-x\right)-c_{A}-\delta \leq 0$, giving $p_{2} \geq$ $N \frac{V_{H}-x-c_{A}-\delta}{V_{H}-x}$. When instead $p_{2}>N \frac{x^{\prime}-x}{V_{H}-x}$, agents $A$ play $L$ if $V_{H}-x^{\prime}-c_{A}-\delta \leq$ 0 , condition always satisfied when $x^{\prime} \geq x_{A}^{D}$. Notice that $N \frac{V_{H}-x-c_{A}-\delta}{V_{H}-x}<N \frac{x^{\prime}-x}{V_{H}-x}$ for $x^{\prime}>x_{A}^{D}$; hence

$$
\bar{r}_{B}(x)=\left\lceil N\left(1-\frac{\delta+c_{A}}{V_{H}-x}\right)\right\rceil .
$$

Analogous considerations can be used to derive $\bar{r}_{A}(x)$. By comparing $\bar{r}_{B}(x)$ with $\bar{r}_{A}(x)$ we get $\bar{r}_{B}(x)<\bar{r}_{A}(x)$ if $x>\widehat{x}$.

Lemma 6 Consider $\Gamma_{U G}$. The minimum number of mutations required to get from $\theta_{x} \in \Sigma_{I H}^{U}$ to an equilibrium which supports an inefficient investment profile are:

$$
\begin{aligned}
& \bar{r}_{A}(x)=\left\lceil N\left(1-\frac{V_{M}-\delta+c_{B}}{x}\right)\right\rceil \text { if } \quad x<\widehat{x} \\
& \bar{r}_{B}(x)=\left\lceil N\left(1-\frac{V_{M}-\delta+c_{A}}{V_{H}-x}\right)\right\rceil \text { if } \quad x>\widehat{x} .
\end{aligned}
$$

Proof. We give the proof for $\bar{r}_{B}(x)$ only. We first derive the mutations required to induce agents to play $L$ when, in $\theta_{x}$, some opponents mutate and play $L$; then we derive the mutations required to induce agents to play $L$ when some opponents mutate by demanding a larger share. 
Consider $\theta_{x} \in \Sigma_{I H}^{U}$ and suppose agents $A$ believe to get the maximum payoff in an $L H$ match and to obtain the minimum payoff in an $H L$ match. Suppose $p_{1}$ agents $B$ mutate by playing $L$; this will induce agents $A$ to play $L$ if $\left(N-p_{1}\right)\left(V_{H}-x-c_{A}-V_{M}+\delta\right)+p_{1}\left(\delta-c_{A}\right) \leq 0$. Solving for $p_{1}$ gives:

$$
r_{B}^{1}(x)=\left\lceil N\left(\frac{V_{H}-x-c_{A}-V_{M}+\delta}{V_{H}-x-\delta-V_{M}+\delta}\right)\right\rceil .
$$

Suppose now $p_{2}$ agents $B$ mutate by claiming $x^{\prime}>x_{A}^{U} \equiv V_{H}-c_{A}-V_{M}+\delta$. Consider the NDG in which agents $B$ either play $x$ or $x^{\prime}>x$ and agents $A$ either play $V_{H}-x$ or $V_{H}-x^{\prime}<V_{H}-x$. A's expected payoff are respectively $\left(V_{H}-x\right) \frac{N-p_{2}}{N}-c_{A}$ and $V_{H}-x^{\prime}-c_{A}$. It thus follows that $A$ 's best reply to $B$ 's mutations is $V_{H}-x$ if $p_{2} \leq N \frac{x^{\prime}-x}{V_{H}-x}$ and it is $V_{H}-x^{\prime}$ otherwise. When $p_{2} \leq N \frac{x^{\prime}-x}{V_{H}-x}, A$ play $L$ if $\frac{N-p_{2}}{N}\left(V_{H}-x\right)-c_{A}-\left(V_{M}-\delta\right) \leq 0$, giving $p_{2} \geq$ $N \frac{V_{H}-x-c_{A}-V_{M}+\delta}{V_{H}-x}$. When instead $p_{2}>N \frac{x^{\prime}-x}{V_{H}-x}, A$ play $L$ if $V_{H}-x^{\prime}-c_{A}-$ $\left(V_{M}-\delta\right) \leq 0$, condition always satisfied when $x^{\prime}>x_{A}^{U}$. We conclude that $N \frac{V_{H}-x-c_{A}-V_{M}+\delta}{V_{H}-x}<N \frac{x^{\prime}-x}{V_{H}-x}$ for $x^{\prime}>x_{A}^{U}$; hence

$$
r_{B}^{2}(x)=\left\lceil N\left(\frac{V_{H}-x-c_{A}-V_{M}+\delta}{V_{H}-x}\right)\right\rceil .
$$

Since $r_{B}^{2}(x)<r_{B}^{1}(x)$ then $\bar{r}_{B}(x)=r_{B}^{2}(x)$. Analogous considerations can be used to derive $\bar{r}_{A}(x)$. By comparing $\bar{r}_{B}(x)$ with $\bar{r}_{A}(x)$ we get $\bar{r}_{B}(x)<\bar{r}_{A}(x)$ if $x>\widehat{x}$

Proof of Proposition 3. Point (a). Consider an equilibrium $\theta_{x} \in \Sigma_{H}^{D}$. The minimum number of mutations required to leave this equilibrium are given in Lemma 11 and Lemma 12. Few computations show that $r_{B}^{+}(x) \leq \bar{r}_{B}(x)$ and $r_{A}^{-}(x) \leq \bar{r}_{A}(x)$. Point (e) of Assumption 1 then implies that $\min \left(r_{B}^{+}(x), r_{A}^{-}(x)\right)>$ 1. Taking point (b) of Proposition 2 into account we conclude that any equilibrium $\theta_{x} \in \Sigma_{H}^{D}$ is locally stable so that $\Sigma_{S}^{D} \subseteq \Sigma_{H}^{D}$. 
Point (b). The proof that $\theta_{x} \in \Sigma_{I H}^{U}$ is locally stable when condition (7) holds true is as for point (a) above. The minimum number of mutations needed to leave this equilibrium are now given by Lemma 11 and Lemma 13. As before, $r_{B}^{+}(x) \leq \bar{r}_{B}(x)$ and $r_{A}^{-}(x) \leq \bar{r}_{A}(x)$; point (e) of Assumption 1 still implies $\min \left(r_{B}^{+}(x), r_{A}^{-}(x)\right)>1$. From point (b) of Proposition 1 we conclude that any equilibrium $\theta_{x} \in \Sigma_{I H}^{U}$ is locally stable so that $\Sigma_{S}^{U} \subseteq \Sigma_{I H}^{U}$. Consider now an equilibrium $\theta_{x} \in \Sigma_{C H}^{U}$ and let condition (7) be satisfied. We show that starting from this equilibrium it is possible to enter the basin of attraction of an equilibrium $\theta \in \Sigma_{L}^{U}$, through a sequence of single-mutation transitions; this, in turn, implies that $\theta_{x} \in \Sigma_{C H}^{U}$ is not locally stable. To see this, let $x \geq x_{A}^{U}$ (the case in which $x \leq x_{B}^{U}$ is symmetric) and let agents' belief drift such that: (i) all agents $A$ expect to get a payoff lower than $c_{A}$ at $H L$ and (ii) all agents $B$ claim $\delta$ at $L H$. Suppose an agent $A$ mutates by playing $L$ and accepting her opponent's demand at $L H$; this mutant thus receives $V_{M}-\delta$. When agents $A$ update, they all imitate and play $L$. When agents $B$ revise they play $L$. The process then reaches a new equilibrium $\theta \in \Sigma_{L}^{U}$.

Point (c). When condition (7) fails, point (b) of Proposition 1 implies that, starting from $\Sigma_{L}^{U} \cup \Sigma_{L H}^{U} \cup \Sigma_{H L}^{U}$, it is possible to enter the basin of attraction of an equilibrium $\theta_{x} \in \Sigma_{C H}^{U}$ through a sequence of single-mutation transitions. From this equilibrium, a new equilibrium $\theta \in \Sigma_{L}^{U}$ is reached by a sequence of single-mutation transitions; this follows from the analysis of the second part of point (b) above which is still valid (when condition (7) fails, we only have to consider that $\left.x_{A}^{U}<x_{B}^{U}\right)$. Consider an equilibrium $\theta \in \Sigma_{L}^{U}$ and suppose agents' belief drift such that: (i) all agents $A$ accept any proposal made by agents $B$ at $L H$ and (ii) all agents $A$ expect to get a share smaller than $c_{A}$ at $H H$. Suppose an agent $B$ mutates by playing $H$ and demanding $x^{*}>c_{B}$ in $L H$. When all agents $B$ update, they choose $H$ since population $A$ has 
accepted her proposal. When agents $A$ revise they continue to play $L$. Hence a new equilibrium $\theta^{\prime} \in \Sigma_{L H}^{U}$ is reached through a sequence of single-mutation transitions (a similar argument can be used to show that from $\theta \in \Sigma_{L}^{U}$ it is possible to reach an equilibrium $\theta^{\prime} \in \Sigma_{H L}^{U}$ ). Collecting all these informations we conclude that the set $\Sigma_{C H}^{U} \cup \Sigma_{L}^{U} \cup \Sigma_{L H}^{U} \cup \Sigma_{H L}^{U}$ is mutation connected. Local stability follows from the observation that, when (7) doesn't hold, one mutation is not enough to leave this mutation connected set.

Before giving the proof of Proposition 4 we briefly summarize the naive minimization test (Binmore at al., 2003) and the radius modified coradius criterion (Ellison, 2000). Let $\theta$ and $\theta^{\prime}$ be two equilibria. The most salient concept is the resistance from $\theta$ to $\theta^{\prime}, r\left(\theta, \theta^{\prime}\right)$; this represents the minimum number of mistakes required to enter $\beta\left(\theta^{\prime}\right)$ starting from $\theta$.

Consider a game with an unperturbed process satisfying the no-cycling condition. In this case for each equilibrium $\theta$ we can draw a tree rooted at $\theta$. This is a direct graph involving all equilibria different from $\theta$ and such that from any equilibrium $\theta^{\prime} \neq \theta$ there is a single path leading to $\theta^{\prime}$. For any tree rooted at $\theta$ we can compute its total resistance by summing all the resistances associated with its edges. The stochastic potential of $\theta$ is the least total resistance among all $\theta$-trees. Young (1993a) proves that an equilibrium is stochastically stable if it has the least stochastic potential among all equilibria. Therefore, to get the stochastically stable equilibrium we must construct the minimum resistance tree. By definition, any transition between equilibria requires at least one mutation. Hence, when constructing a minimum resistance tree, we can ignore all equilibria that are not locally stable. The minimum resistance tree can be found by applying the naive minimization test and it works as follows. Take any locally stable equilibrium and join it to the locally stable equilibrium which can be reached with the minimum number of mutations. Suppose this 
generates a directed graph with a single cycle and this cycle contains the edge with the largest resistance. By removing that edge we obtain a tree rooted at the equilibrium that was the origin of the edge. This is the minimum resistance tree and its root is the only stochastically stable equilibrium of the game.

Consider now a game with an unperturbed process which does not satisfy the no-cycling condition. In this case we can only hope that the sufficient condition for stochastic stability developed by Ellison (2000) work. Consider a union of absorbing sets, $\Sigma^{\prime}$. The Radius $R\left(\Sigma^{\prime}\right)$ is the minimum number of mutations needed to escape from $\beta\left(\Sigma^{\prime}\right)$ and enter another one with positive probability. Consider an arbitrary state $\theta \notin \Sigma^{\prime}$ and let $\left(m_{1}, m_{2}, . ., m_{T}\right)$ be a path from $\theta$ to $\Sigma^{\prime}$ where $\Omega_{1}, \Omega_{2}, \ldots \Omega_{r}$ is the sequence of absorbing sets through which the path passes consecutively. Obviously $\Omega_{i} \notin \Sigma^{\prime}$ for $i<r$ and $\Omega_{r} \subset \Sigma^{\prime}$. Notice that an absorbing set can appear several times in this sequence but not consecutively. The modified cost of this path is defined by:

$$
c^{*}\left(m_{1}, \ldots, m_{T}\right)=c\left(m_{1}, . ., m_{T}\right)-\sum_{i=2}^{r-1} R\left(\Omega_{i}\right)
$$

where $c\left(m_{1}, . ., m_{T}\right)$ is the total number of mutations over the path $\left(\theta, m_{1}, m_{2}, . ., m_{T}\right)$. Let $c^{*}\left(\theta, \Sigma^{\prime}\right)$ be the minimal modified cost among all paths from $\theta$ to $\Sigma^{\prime}$. The Modified Coradius of $\beta\left(\Sigma^{\prime}\right)$ is then:

$$
C R^{*}\left(\Sigma^{\prime}\right)=\max _{\theta \notin \Sigma} c^{*}\left(\theta, \Sigma^{\prime}\right)
$$

Theorem 2 of Ellison (2000) shows that every union of absorbing sets $\Sigma^{\prime}$ with $R\left(\Sigma^{\prime}\right)>C R^{*}\left(\Sigma^{\prime}\right)$ encompasses all the stochastically stable states.

Proof of Proposition 4. Point (a) follows by applying the naive minimization test to the set of locally stable equilibria $\Sigma_{H}^{D}$. Consider Lemmas 4 and 5; we already know that $r_{B}^{+}(x) \leq \bar{r}_{B}(x)$ and $r_{A}^{-}(x) \leq \bar{r}_{A}(x)$. Consider an 
equilibrium $\theta_{x}$ with $x<\widehat{x}$; we know from Lemma 4 that $r_{B}^{+}(x)<r_{A}^{-}(x)$ so that the least resistance path out of $\theta_{x}$ is toward $\theta_{x+\delta}$. Consider now an equilibrium $\theta_{x}$ with $x>\widehat{x}$; we know by Lemma 4 that $r_{A}^{-}(x)<r_{B}^{+}(x)$ so that the least resistance path is toward $\theta_{x-\delta}$. Since $r_{B}^{+}(x)$ is a strictly increasing function of $x$ and $r_{A}^{-}(x)$ is a strictly decreasing function of $x$, at $\theta_{\widehat{x}}$ we get

$$
r_{B}^{+}(\widehat{x}-\delta)<r_{B}^{+}(\widehat{x})=r_{A}^{-}(\widehat{x})>r_{A}^{-}(\widehat{x}+\delta)
$$

Hence, for each equilibrium $\theta_{x}$, the naive minimization test produces a set of edges pointing to $\theta_{x+\delta}$ if $x<\widehat{x}$ and to $\theta_{x-\delta}$ if $x>\widehat{x}$. Moreover there is a directed graph with a single cycle formed by the edge pointing from $\theta_{\widehat{x}}$ to $\theta_{\widehat{x}+\delta}$ (or to $\theta_{\widehat{x}-\delta}$ ) and the edge going to the opposite direction. By removing the edge with the highest resistance (i.e. $\theta_{\widehat{x}} \rightarrow \theta_{\widehat{x}+\delta}$ ) we get the minimum resistance tree, which is rooted at $\theta_{\widehat{x}}$. The unique stochastically stable outcome is thus $\left(H H, V_{H} / 2, V_{H} / 2\right)$.

Point (b). Let (7) be satisfied so that the set $\Sigma_{I H}^{U}$ is well defined. Let us fix an equilibrium $\theta_{x} \in \Sigma_{I H}^{U}$. We know from point (b) of Proposition 1 that $c^{*}\left(\theta, \theta_{x}\right)=1$ for any equilibrium $\theta \in \Sigma_{L H}^{U} \cup \Sigma_{H L}^{U} \cup \Sigma_{L}^{U}$ as well as $c^{*}\left(\Omega, \theta_{x}\right)=1$ for any $\Omega \in \Omega_{c}$. Hence, the minimal modified cost among all paths from an absorbing set outside $\Sigma_{I H}^{U}$ to any equilibrium $\theta_{x}$ is equal to 1 . We now derive the equilibrium $\theta_{x}$ compatible with the radius modified coradius criterion. In order to derive $R\left(\theta_{x}\right)$ we use Lemmas 4 and 6 . We already know that $r_{B}^{+}(x) \leq \bar{r}_{B}(x)$ and $r_{A}^{-}(x) \leq \bar{r}_{A}(x)$. Therefore we conclude that $R\left(\theta_{x}\right)=r_{B}^{+}(x)$ if $x<\widehat{x}$ while $R\left(\theta_{x}\right)=r_{A}^{-}(x)$ if $x \geq \widehat{x}$. Recall that $r_{B}^{+}(x)$ (resp. $\left.r_{A}^{-}(x)\right)$ is an increasing (resp. decreasing) function. Two cases must be considered, both compatible with condition (7).

In the first case $x_{B}^{U}+\delta<\widehat{x} \leq x_{A}^{U}-\delta$; this occurs when $c_{A} \leq \frac{V_{H}}{2}-V_{M}$. Consider the equilibrium $\theta_{\widehat{x}}$ and let $\theta_{x}$ be a different equilibrium. From Lemma 
4 we know that $c^{*}\left(\theta_{x}, \theta_{\widehat{x}}\right)=r_{B}^{+}(x)$ if $x_{B}^{U}+\delta \leq x<\widehat{x}$ and $c^{*}\left(\theta_{x}, \theta_{\widehat{x}}\right)=r_{A}^{-}(x)$ if $\widehat{x}<x \leq x_{A}^{U}-\delta$. Therefore $C R^{*}\left(\theta_{\widehat{x}}\right)=\max \left(r_{B}^{+}(\widehat{x}-\delta), r_{A}^{-}(\widehat{x}+\delta)\right)$. Of course, $R\left(\theta_{\widehat{x}}\right)=r_{B}^{+}(\widehat{x})=r_{A}^{-}(\widehat{x})$. Monotonicity implies $R\left(\theta_{\widehat{x}}\right)>C R^{*}\left(\theta_{\widehat{x}}\right)$; it then follows from Theorem 2 of Ellison (2000) that the only stochastically stable outcome is $\left(H H, V_{H} / 2, V_{H} / 2\right)$.

In the second case $x_{B}^{U}+\delta<x_{A}^{U}-\delta<\widehat{x}$; this occurs when. $\frac{V_{H}}{2}-V_{M}<$ $c_{A} \leq V_{H}-2 V_{M}-c_{B}$. Let $\widetilde{x} \equiv x_{A}^{U}-\delta$. Consider the equilibrium $\theta_{\widetilde{x}}$ and let $\theta_{x}$ be a different equilibrium. For any $x \in\left[x_{B}^{U}+\delta, \widetilde{x}\right)$, we know from Lemma 4 that $c^{*}\left(\theta_{x}, \theta_{\widetilde{x}}\right)=r_{B}^{+}(x)$. The monotonicity of $r_{B}^{+}(x)$ implies that $C R^{*}\left(\theta_{\widetilde{x}}\right)=r_{B}^{+}(\widetilde{x}-\delta)$. Since $R\left(\theta_{\widetilde{x}}\right)=r_{B}^{+}(\widetilde{x})$, we get $R\left(\theta_{\widetilde{x}}\right)>C R^{*}\left(\theta_{\widetilde{x}}\right)$; it follows from Ellison (2000) that the only stochastically stable outcome is $\left(H H, V_{H}-\left(x_{A}^{U}-\delta\right), x_{A}^{U}-\delta\right)$ and the distributional norm is $\left(V_{M}+c_{A}, V_{H}-V_{M}-c_{A}\right)$. 
Acknowledgement 1 This paper was written while Lidia Bagnoli was research fellow of the Department of Economics, University of Bologna; financial support from the Department of Economics gratefully acknowledged. The paper has been presented at seminars held at the University of Bologna and at the Catholic University of Milan, at the workshop on Social Norms and Social Preferences (Trento, 2013) and at the ISNIE conference (Florence, 2013). We thank all participants for their comments. We would like to express our gratitude to two anonymous referees for their detailed and useful observations on a previous version of the paper. We are the only responsible for errors and imprecisions. 


\section{References}

[1] Alvard S. M., Nolin D. A., 2002. Rousseau's whale hunt ?. Current Anthropology 43, 533-559.

[2] Andreozzi L., 2010. An evolutionary theory of social justice: choosing the right game. European Journal of Political Economy 26, 320-329.

[3] Andreozzi L., 2012. Property rights and investment: an evolutionary approach. Games and Economic Behavior, 74, 1-11.

[4] Barling B., von Siemens F., 2010. Equal sharing rules in partnership. Journal of Institutional and Theoretical Economics 166, 299-320.

[5] Bell D., 1995. On the nature of sharing: beyond the range of methodological individualism. Current Anthropology, 36, 826-830.

[6] Binmore K., Samuelson L., Young P., 2003. Equilibrium selection in bargaining models. Games and Economic Behavior 45, 296-328.

[7] Boehm C., 2004. What makes humans economically distinctive ? A threespecies evolutionary comparison and historical analysis. Journal of Bioeconomics $6,109-135$.

[8] Boehm C., 2012. Moral Origins. The Evolution of Virtue, Altruism, and Shame. Basic Books, New York.

[9] Dawid H., MacLeod B., 2001. Hold up and the evolution of bargaining conventions. European Journal of Economic and Social Systems 15, 139169.

[10] Dawid H., MacLeod B., 2008. Hold-up and the evolution of investment and bargaining norms. Games and Economic Behavior 62, 26-52. 
[11] Ellingsen T., Robles J., 2002. Does evolution solve the hold-up problem ?. Games and Economic Behavior 39, 28-53.

[12] Ellison G., 2000. Basin of attraction, long-run stochastic stability, and the speed of step-by-step evolution. Review of Economic Studies, 67, 17-45.

[13] Foster D., and Young P., 1990. Stochastic evolutionary game dynamics. Theoretical Population Biology, 38, 219-232.

[14] Hill K., 2002. Altruistic cooperation during foraging by the Ache, and the evolved human predisposition to cooperate. Human Nature 13, 105-128.

[15] Noldeke G., Samuelson L., 1993. An evolutionary analysis of backward and forward induction. Games and Economic Behavior 5, 425-454.

[16] Samuelson L., 1997. Evolutionary Games and Equilibrium Selection. MIT Press, Cambridge Mass.

[17] Troger T., 2002. Why sunk costs matter for bargaining outcomes: an evolutionary approach. Journal of Economic Theory 102, 375-402.

[18] Woodburn J., 1982. Egalitarian Societies. Man 17, 431-451.

[19] Young P., 1993. An evolutionary model of bargaining. Journal of Economic Theory 59, 145-168.

[20] Young P., 1993a. The evolution of conventions. Econometrica 61, 57-84. 


\section{Figures captions}

Figure 1: When one agent only invests, a small pie valued 40 is produced; investment cost is 20 for $A$ and 0 for $B$. The three decreasing lines of dots represent (feasible) payoff profiles in the case in which both $A$ and $B$ invest (upper line), only $B$ invests (middle line) and only $A$ invests (lower line). To each of these decreasing lines there corresponds a given gross surplus. For any payoff pair resulting from a division of this gross surplus, the sum of the payoff gives the net surplus. The upper increasing line represents the payoff pair corresponding to the equal division of the gross surplus; the lower increasing line represents the gross surplus divisions giving rise to payoff equality. The dotted square represents the set of payoff such that both agents get at least 40 (this matters for $\Gamma_{U G}$ only since it represents the maximum payoff an agent can hope to obtain by not investing). The big dot represents the payoff profile corresponding to the stochastically stable equilibrium. Stochastic stability selects equal division of the efficient gross surplus in $\Gamma_{D G}$ but not in $\Gamma_{U G}$ where instead the less efficient agent $A$ receives more than half of the efficient gross surplus.

Figure 2: The Figure illustrates Proposition 6 . In $\Gamma_{D G}$ the equilibria $\theta_{x}$ are stochastically stable in region $A D E$. In $\Gamma_{U G}$ the equilibria $\theta_{x}$ are stochastically stable in region $A C F$; no norms evolve in region $C D E F$ since several outcomes are stochastically stable. 


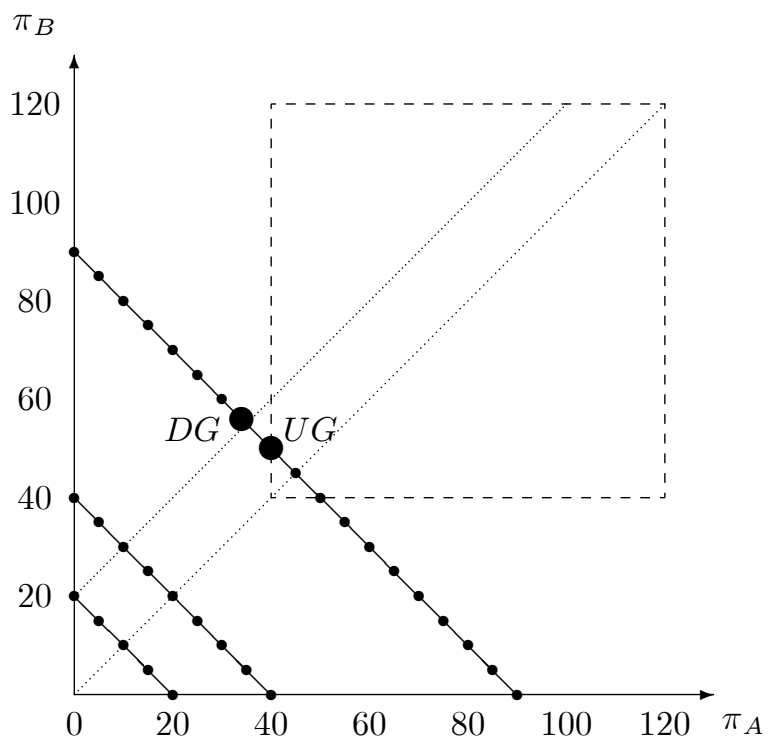

Figure 1 


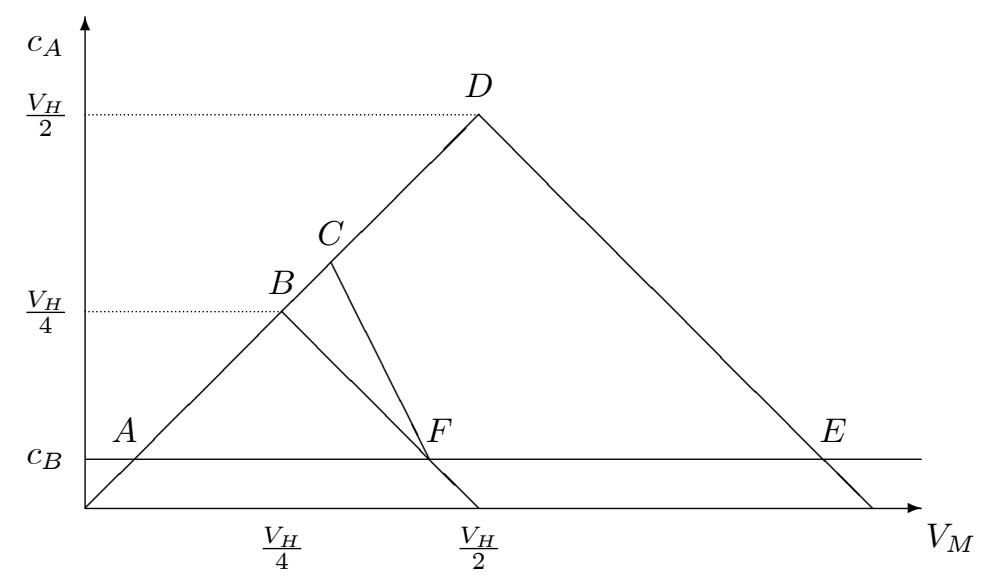

Figure 2 